\title{
Unified Grey Relational Analysis on Transformer DGA Fault Diagnosis
}

\author{
Wang Qizhi ${ }^{*}, 1$ and Wang Xiaoxia ${ }^{2}$ \\ ${ }^{I}$ College of Mechanical Engineering and Automation, Huaqiao University, Jimei Street 668, Xiamen, Fujian, 361021, \\ China \\ ${ }^{2}$ College of Computer Science \& Technology, Huaqiao University, Jimei Street 668, Xiamen, Fujian, 361021, China
}

\begin{abstract}
Power transformer is one of the most important equipments in the electric system. Dissolved Gases Analysis is an important method in the diagnosis of internal fault of transformers. Because the signs and types of fault have complicated nonlinear relations, the traditional methods cannot exactly meet the requirements of engineer application. This paper proposes synthesis of relational grey incidence to analyze the comparability and the proximity of the sequence which can effectively diagnose the type of transformer faults.
\end{abstract}

Keywords: Dissolved gases of power transformer, Unified relational grey analysis, DGA technique, Grey relational analysis.

\section{INTRODUCTION}

Fault Power transformer is important equipment in the network between the power generator and power transmission [1-4]. It is a major apparatus in the power system and its correct functioning is important to system operation. In order to achieve better service quality, it is essential to routinely examine the abnormal condition and avoid interrupting power service.

DGA technique is widely applied to detect internal faults under uninterrupted power service. Available gases from chromatographic analysis of the insulation oil can contain concentrations of dissolved $\mathrm{H}_{2}, \mathrm{CH}_{4}, \mathrm{C}_{2} \mathrm{H}_{2}, \mathrm{C}_{2} \mathrm{H}_{4}$ and $\mathrm{C}_{2} \mathrm{H}_{6}$.

A grey system is defined as a system where "partial information is known, while some information is unknown". It is a practical technique to deal with the problem, which is characterized by uncertainty and has poor or incomplete information. Transformer operation is a complex system (i.e., grey system). Fault diagnosis, which considers uncertainties as grey value, uses the limited known information. Forecast judgments and decisions are exploited by information processing. The grey relation analysis is to analyze uncertain relations between a major factor and other factors in a given system.

\section{GREY RELATIONAL ANALYSIS}

The grey relational analysis is applicable to analyze uncertain relations; it has a small amount of calculation, which is very convenient. It is a practical technique which is characterized by uncertainty or incomplete information [5-9]. Traditional GRA is based on the geometric relation between sequence data in the relational space. The grey relational

*Address correspondence to this author at the College of Mechanical Engineering and Automation, Huaqiao University, Jimei Street 668, Xiamen, Fujian, 361021, China; Tel:13655991726; Fax: 0595-22866969; E-mail:wangqz@hqu.edu.cn grade is derived by the obtained grey relational coefficients. Transformer operation is a complex system (i.e., grey system). Its fault diagnosis considers uncertainties as grey value and subsequently uses the limited information which is known. Its forecast and judgment decisions are determined by information processing. The grey relational grade between two series at a certain point is called the grey relational coefficient.

GRA (grey relational analysis) is an analysis of the geometric proximity between different discrete sequences within a system. The closer the curves, the higher the associated degree between the corresponding sequences. It also describes the process of system development factors of relative changes.

The basic idea of the method of grey relational grade analysis is based on the similarity of the geometry of sequence curves. If its relation is close, the curve is closer, and the associated degree between the corresponding sequences is higher, otherwise it is lower. It also describes the process of system development factors of relative changes. If the two relative changes in the development process are basically consistent, their relevance is great; on the contrary, their correlation is small.

Slope grey relational analysis (SGRA) reflects the rate of change of the sequence and trends; while the area grey relational analysis (AGRA) calculates the correlation degree between the two curves using the related area between the curves. If the area is larger, the degree of correlation is smaller, which reflects the degree of dispersion curves. There are two complementary roles. If the SGRA and AGRA are both considered, then a more comprehensive description is obtained between the two sequences tightness.

\subsection{The Analysis Method of Slope Grey Relational Analysis (SGRA)}

Using slope relational degree analysis method, the parameter sequence $i=0,1,2, \ldots, \mathrm{Q}$. 
$X_{i}=\left(x_{i}(1), x_{i}(2), \ldots, x_{i}(n)\right), \quad \mathrm{i}=0,1,2, \ldots, \mathrm{q}$

Comparative sequence.

Defined $\xi_{m}(t)$ correlation coefficient with the grey of $X_{i}$ and $Y_{j}$ is known as the correlation coefficient with the grey slope.

$$
\xi_{m}(t)=\frac{1+\left|\frac{1}{\bar{x}_{i}} \bullet \frac{\Delta x_{i}(t)}{\Delta t}\right|}{1+\left|\frac{1}{\bar{x}_{i}} \bullet \frac{\Delta x_{i}(t)}{\Delta t}\right|+\left|\frac{1}{\bar{x}_{i}} \bullet \frac{\Delta x_{i}(t)}{\Delta t}-\frac{1}{y_{i}} \bullet \frac{\Delta y_{i}(t)}{\Delta t}\right|}
$$

Defining the degree grey slope incidence

$\varepsilon_{j i}=\left(\sum_{t=1}^{n-1} \xi_{m}(t)\right) /(n-1)$

\subsection{Area Grey Relational Analysis (AGRA)}

Setting reference sequence $Y_{i}=\left(y_{i}(1), y_{i}(2), \cdots, y_{i}(n)\right)$, comparative sequence,

$X_{i}^{\prime}=\left(x_{i}^{\prime}(1), x_{i}^{\prime}(2), \cdots, x_{i}^{\prime}(n)\right), i=1,2, \cdots, m$

$x_{i}=x_{i}^{\prime}(i)-\left(x_{i}(1)-y_{i}(1)\right)$

AGRA calculates the correlation degree according to the area between $X_{i}$ and $Y_{i}$.

Area under the curve and the correlation calculate the area between the correlation degrees. Obviously, the smaller the area, the closer the curve, and the greater the degree of association; conversely, the larger the area, the smaller the correlation.

(1) If the curves do not intersect, it can be seen as a trapezoid area

$s_{i k}=\frac{1}{2} *\left[\left|x_{i}(k)-x_{0}(k)\right|+\left|x_{i}(k+1)-x_{0}(k+1)\right|\right]$

(2) If the curves intersect, it can be seen as a superposition of two triangular areas.

$s_{i k}=\frac{1}{2} * \frac{\left[x_{i}(k+1)-x_{0}(k+1)\right]^{2}+\left[x_{i}(k)-x_{0}(k)\right]^{2}}{\left|x_{i}(k+1)-x_{0}(k+1)\right|+\left|x_{i}(k)-x_{0}(k)\right|}$

$\delta\left(X_{0}\right)=\frac{L_{0}}{\bar{K}_{0}} \times 100 \%$

where

$$
\begin{aligned}
& \bar{K}_{0}=\frac{1}{n-1} \sum_{j=1}^{n-1} S_{j} \\
& L_{0}=\sqrt{\frac{1}{n-2} \sum_{j=1}^{n-1}\left(S_{j}-\bar{K}_{0}\right)^{2}} \\
& \gamma\left(X_{0}, X_{i}\right)=\operatorname{sgn}\left(K_{0 i}\right) \frac{1}{1+\left|\delta\left(X_{0}\right)\right|}
\end{aligned}
$$

$\gamma\left(X_{i}, Y_{i}\right)=\left\{\begin{array}{c}\frac{1}{1+\delta\left(X_{0}\right)^{2}}, K_{0 i} \geq 0 \\ -\frac{1}{1+\delta\left(X_{0}\right)^{2}}, K_{0 i} \prec 0\end{array}\right.$

\subsection{Unified grey Relational Analysis (UGRA) Consider $\mathbf{0}<\varepsilon<\mathbf{a}, \mathbf{0}<\gamma<\mathbf{1}$}

Defined

$\lambda=\sqrt{\frac{\varepsilon^{2}+\gamma^{2}}{2}}$

where $\varepsilon$ is SGAR; $\gamma$ is AGAR

Character $0<\lambda<1$

\section{FAULT DIAGNOSIS OF THE GRAY CORRELAT- ION ANALYSIS METHOD AND STEPS IN TRANS- FORMER INTERNAL}

DGA is widely used by utilities for monitoring the health of oil filled transformers.

Vector state model's multiple characteristic parameters of transformer equipment status are mainly used in the internal fault diagnosis; Compared to the degree of association examination pattern vector and the standard mode of the state vector, the standard model state can be determined.

Set five kinds of typical oil dissolved gases $\mathrm{H}_{2}, \mathrm{CH}_{4}$, $\mathrm{C}_{2} \mathrm{H}_{4}, \mathrm{C}_{2} \mathrm{H}_{6}$ and $\mathrm{C}_{2} \mathrm{H}_{2}$ as the characteristic parameters, the state pattern vector (AUTHOR: The highlighted sentence is incomplete)

Five typical oil dissolved gases $\mathrm{H}_{2}, \mathrm{CH}_{4}, \mathrm{C}_{2} \mathrm{H}_{4}, \mathrm{C}_{2} \mathrm{H}_{6}$ and $\mathrm{C}_{2} \mathrm{H}_{2}$ as characteristic parameters are selected.

The relationship between the faults of transformer internal fault detection results are analyzed. There are 9 standard state pattern vectors: $\mathrm{x}_{1}$ is the normal operation mode; $x_{2}$ is the failure mode for the low energy discharge; $x_{3}$ is the high-energy discharge, $\mathrm{x}_{4}$ is the failure mode for the low temperature overheating; $\mathrm{x}_{5}$ is the high temperature overheating fault model for failure mode; $\mathrm{x}_{6}$ is the screen's discharge; $x_{7}$ is the inter laminar failure mode; $x_{8}$ is the tapchanger fault mode; and $\mathrm{x}_{9}$ is the core two-point or multipoint grounding failure mode. The characteristics of the fault model vector matrix:

$$
X=\left(\begin{array}{c}
x_{1} \\
x_{2} \\
\vdots \\
x_{9}
\end{array}\right)=\left(\begin{array}{ccccc}
x_{1}(1) & x_{1}(2) & x_{1}(3) & x_{1}(4) & x_{1}(5) \\
x_{2}(1) & x_{2}(2) & x_{2}(3) & x_{2}(4) & x_{2}(5) \\
\vdots & \vdots & \vdots & \vdots & \vdots \\
x_{9}(1) & x_{9}(2) & x_{9}(3) & x_{9}(4) & x_{9}(5)
\end{array}\right)
$$

Through the transformer fault oil dissolved gas analysis (DGA), and by the large amounts of data tests, the standard transformer internal fault modes are finally obtained. The standard spectrum used in transformer fault diagnose is shown in Table 1.

From Table 2, it can be found that the diagnosis of UGAR is more accurate than the method of three ratios of typical transformer fault diagnosis, because it is close to the real fault. 
Table 1. Normative mode of transformer fault.

\begin{tabular}{|c|c|c|c|c|c|}
\hline$x_{i}(1)$ & $x_{i}(1)\left(H_{2}\right) \%$ & $x_{i}(2)\left(\mathrm{CH}_{4}\right) \%$ & $x_{i}(3)\left(C_{2} H_{4}\right) \%$ & $x_{i}(4)\left(C_{2} H_{2}\right) \%$ & $x_{i}(5)\left(C_{2} H_{6}\right) \%$ \\
\hline$x_{1}$ & 46.1 & 21.5 & 15.8 & 1.2 & 61.5 \\
\hline$x_{2}$ & 58 & 44.9 & 20.6 & 23.5 & 11 \\
\hline$x_{3}$ & 43.7 & 30.2 & 46.6 & 19.4 & 3.7 \\
\hline$x_{4}$ & 15.3 & 26.2 & 52.8 & 0 & 21 \\
\hline$x_{5}$ & 11.3 & 24.6 & 59.9 & 2.8 & 12.7 \\
\hline$x_{6}$ & 58.6 & 30.5 & 26.2 & 38.4 & 4.9 \\
\hline$x_{7}$ & 28.8 & 28.2 & 34.4 & 33.4 & 3.9 \\
\hline$x_{8}$ & 13.6 & 21.6 & 58.1 & 9.5 & 10.8 \\
\hline$x_{9}$ & 11.2 & 30.8 & 56.2 & 1.4 & 11.6 \\
\hline
\end{tabular}

Table 2. The diagnosis of representative fault in power transformer.

\begin{tabular}{|c|c|c|c|c|c|c|c|c|}
\hline Serial & $\begin{array}{c}\text { Three Ratios } \\
\text { of Typical Transformer } \\
\text { Fault Diagnosis }\end{array}$ & $\begin{array}{c}\mathbf{H}_{2} / \\
\mathbf{p p m}\end{array}$ & $\begin{array}{c}\mathbf{C H} / / \\
\mathbf{p p m}\end{array}$ & $\begin{array}{c}\mathbf{C}_{2} \mathbf{H}_{4} / \\
\mathbf{p p m}\end{array}$ & $\begin{array}{c}\mathbf{C}_{2} \mathbf{H}_{2} / \\
\mathbf{p p m}\end{array}$ & $\begin{array}{c}\mathbf{C}_{2} \mathbf{H}_{6} / \\
\mathbf{p p m}\end{array}$ & $\begin{array}{c}\text { Three Ratios } \\
\text { of Typical Transformer } \\
\text { Fault Diagnosis }\end{array}$ & $\begin{array}{c}\text { UGAR } \\
\mathbf{R e a l} \text { Fault }\end{array}$ \\
\hline \hline 1 & $\begin{array}{c}\text { Taikang Station } \\
\text { No. 1, main transformer }\end{array}$ & 164 & 244 & 103 & 497 & 8.3 & $\begin{array}{c}\text { High temperature } \\
\text { overheating fault model temperature } \\
\text { overheating } \\
\text { fault model }\end{array}$ \\
\hline 2 & $\begin{array}{c}\text { Main transformer station } \\
7 \#\end{array}$ & 70.4 & 69.5 & 241.2 & 10.4 & 28.9 & $\begin{array}{c}\text { High temperature } \\
\text { overheating fault } \\
\text { model }\end{array}$ \\
\hline
\end{tabular}

\section{CONCLUSION}

This paper presented a comprehensive approach to calculate gray correlation analysis between the sequence similarity and proximity. In this paper, the UGRA was adopted for fault diagnosis of faults for power transformers. Through examples, it has been proven that this method can effectively diagnose transformer internal fault type, which is consistent with the actual. Since the power transformer fault diagnosis based on the UGRA classifier needs few samples and training time and has good reliability, it is very suitable for fault diagnosis of transformer.

\section{CONFLICT OF INTEREST}

The authors confirm that this article content has no conflict of interest.

\section{ACKNOWLEDGEMENTS}

Declared none.

\section{REFERENCES}

[1] R. Liao, H. Zheng, S. Grzybowski, and L. Yang, "Particle swarm optimization-least squares support vector regression based forecasting modelon dissolved gases in oil-filled power transformers", Electric Power Systems Research, vol. 81, pp. 2074-2080, 2011.

[2] M. Jianbing, and X. Dong, "Mathematical modeling of heat flux distribution of plasma arc by transverse a lternating magnetic field", The Open Mechanical Engineering Journal, vol. 5, pp. 1-8, 2013.

[3] Z. Guo, C. Yuan, and P. Liu, "Study on identification model of cylinder liner-piston ring using vibration analysis based on fuzzy cmeans clustering", The Open Mechanical Engineering Journal, vol. 12, pp. 126-132, 2013.

[4] L. Jiang, "Gas path fault diagnosis system of aero-engine based on Grey Relationship Degree", Procedia Engineering, vol. 15, pp. 4774-4779, 2011.

[5] C.-H. Lin, C.-H. Wu, and P.-Z. Huang, "Grey clustering analysis for incipient fault diagnosis in oil-immersed transformers", Expert Systems with Applications, vol. 36, pp.1371-1379, 2009.

[6] Y.-H. Lin, P. -C. Lee, and T.-P. Chang, "Practical expert diagnosis model based on the grey relational analysis technique", Expert Systems with Applications, vol. 36, pp. 1523-1528, 2009.

[7] Z. YanPing, H. ShuHong, H. JingHong, S. Tao, and L. Wei, "Continuous wavelet grey moment approach for vibration analysis of rotating machinery", Mechanical Systems and Signal Processing, vol. 20, pp. 1202-1220, 2006.

[8] C.-I. Chen, and S.-J. Huang, "The necessary and sufficient condition for $\mathrm{GM}(1,1)$ grey prediction model", Applied Mathematics and Computation, vol. 219, pp. 6152-6162, 2013.

[9] N. M. Xie, and S. F. Liu, "Discrete grey forecasting model and its optimization", Applied Mathematical Modelling, vol. 33, pp.11731186, 2009.

Received: January 17, 2014

(C) Wang Qizhi and Wang Xiaoxia.; Licensee Bentham Open.

This is an open access article licensed under the terms of the Creative Commons Attribution Non-Commercial License (http://creativecommons.org/licenses/ by-nc/4.0/) which permits unrestricted, non-commercial use, distribution and reproduction in any medium, provided the work is properly cited. 